\title{
Stérilisation opératoire et maladie mentale. Une étude de cas
}

par Philippe Ehrenström

\section{RÉsumé}

Examinant le cas vaudois (1928), on constate que la stérilisation forcée des malades mentaux reposait sur la peur d'une "chute génétique» et sur des considérations sur le coût de l'assistance aux moins favorisés. Propre aux malades mentaux, la «menace héréditaire» était comprise comme sapant l'ordre biologique, économique et social de la société. Ce sont les plus pauvres et les moins considérés qui ont été les principales victimes de cette mesure.

\section{Introduction}

La stérilisation légale des malades mentaux est un thème dont les fortunes dépassent de très loin les frontières du canton de Vaud. La mesure est enfant des Amériques, où on a légiféré depuis 1907. D’autres Etats suivront: le canton de Vaud en 1928, le Danemark en 1929, la Norvège en 1934, la Suède et la Finlande en 1935, l'Estonie en 1937. Légiféreront aussi la Lettonie, l'Allemagne et le Japon ${ }^{1}$. Quantitativement, certains Etats ont opéré de manière industrielle: il y aura environs 300 interventions déclarées dans le canton de Vaud entre 1919 et 1944, contre 4600 en Californie pour les seules années 1917-1925.

L'article $28^{\text {bis }}$ de la loi vaudoise sur le régime des personnes atteintes de maladie mentale, voté en 1928 et situé dans les «mesures de préservation», ne peut donc se prévaloir d'aucune originalité ou antériorité ${ }^{2}$. On aura soin d'ajouter que l'opération était pratiquée bien avant sa légalisation, dès 1892 à Zurich, dès 1918 dans le canton de Vaud.

Cet épisode, plutôt triste, de l'histoire médicale en Suisse, reste cependant un exemple intéressant pour l'étude des idées et de leur circulation entre le monde politique, les médecins et l'opinion. Il introduira le lecteur à des évolutions et à des tendances multiformes, du social-darwinisme naïf de certains psychiatres au fonctionnalisme déjà désincarné de l'administration de la santé. 


\section{Statut et images du malade mental}

L'exception ou la dérogation à la norme, en droit, se fonde souvent sur la notion de risque, associée à celles de protection et de coercition. L'état d'urgence, par exemple, ne peut être décrété en théorie que quand s'exercent des forces exceptionnelles qui mettent en péril la sécurité externe ou interne d'un pays, le fonctionnement normal de ses institutions. Des forces dérogatives, libérées par les mesures de mise en exception propres au pouvoir souverain, peuvent alors entrer en action : gouvernement par décret, censure des libertés... Le raisonnement est le même quand il s'agit de déroger aux principes qui régissent l'exercice de la liberté individuelle lors de pandémies ou de régler l'internement légal et obligatoire des alcooliques.

Les corollaires obligés des rapports du risque et de la maladie, la protection du bien commun et la coercition, se retrouvent dans les différentes moutures et révisions de la loi sur le régime des personnes atteintes de maladie mentale, votée par le Grand Conseil vaudois en $1901^{3}$.

Ce texte premier posait les principes suivants: la loi protège tout d'abord le malade des risques qu'il fait courir à lui-même quand son état nécessite des soins. La protection s'étend ensuite à tout tiers contre les risques que présente le malade pour autrui. La loi satisfait cette double opération en énonçant des mesures appropriées, au premier rang desquelles l'internement, très sévèrement réglementé. Le Grand Conseil est représenté dans tout ce qui concerne l'application de la loi par une autorité administrative, le Conseil de santé, qui bénéficie de pouvoirs étendus.

La révision de 1921 innove en élargissant les catégories concernées par les textes aux toxicomanes. Leur inclusion dans un texte sur les maladies mentales ne doit pas surprendre. La toxicomanie est effectivement très souvent affiliée dans les sources à des troubles psychiques, physiques ou moraux qui en constitueraient la cause effective ou l'effet ${ }^{4}$.

Tout change en 1928. Les textes reprennent la notion de risque, mais en y superposant d'autres sens. La nouveauté, c'est que l'on envisage le malade mental comme un être nuisible, anti-social et dangereux pour les qualités génétiques du corps social. Il y a menace donc, à la fois pour l'ordre public et pour la sécurité biologique. Ce qui intéresse le législateur, c'est la possibilité que le malade transmette par la procréation une tare indéfinie à sa descendance:

«Lorsqu'il est à prévoir qu'une personne atteinte de maladie mentale ou d'une infirmité mentale reconnue incurable ne peut avoir qu'une descendance tarée, cette personne peut être l'objet de mesures d'ordre médical pour empêcher la survenance d'enfants.. ${ }^{5}$ 
Les débats montrent clairement que l'on a procédé avec la volonté avouée de préserver les qualités physiques et psychiques de la population, ce que la commission législative appelait la "préservation de la race». Une autre prose, limpide et de même origine, utilisait ainsi avec une rare élégance le terme de «déchets», dont il importait bien sûr de limiter par la stérilisation les méfaits. L'idée même de la négativité sociale et raciale du malade mental traverse toutes les interventions ${ }^{6}$.

Car que craint-on? Les délinquants sexuels, les criminels récidivistes, les pervers? Non, parce qu'ils sont «traités» sous les dispositions du Code Pénal, «accommodés» par les pratiques en vigueur de la médecine légale, castrés parfois ${ }^{7}$. Le texte de 1928 ne les concerne pas. On est ici très loin des textes américains qui incluaient délinquance et maladie dans les mêmes dispositions ${ }^{8}$.

C'est un autre type de menace que l'on veut combattre, l'effrayante multiplication d'individus dont les «tares héréditaires» induisent des comportements «anti-sociaux». J'ai montré ailleurs ce qu'il fallait entendre par ces termes, la poursuite de comportements et de modes de vie marginaux que l'on jugeait révélateurs d'un trouble pathologique ${ }^{9}$. Qu'il me suffise de rappeler la vigueur avec laquelle on prophétisait le spectre de la chute génétique. Pour rester dans le canton de Vaud, le docteur Mahaim, de Cery, parlait de «danger public» en évoquant les descendances indésirables des malades mentaux. Auguste Forel proposait l'euthanasie dans certains cas. Le docteur Boven évoquait les méfaits d'«existences néfastes» qu'il ne fallait pas hésiter à retrancher ${ }^{10}$.

\section{Eléments de structure}

L'exposé des motifs par la commission législative reprend très clairement le thème de la menace contre le corps social, en incluant le malade ou le toxicomane dans un ensemble plus vaste qui comprend tous les motifs possibles d'affaiblissement de la communauté. C'est la raison pour laquelle l'article $28^{\text {bis }}$ sur la stérilisation des malades mentaux s'insère dans un dessein plus vaste, celui de la réforme de la loi sur l'organisation sanitaire de 1897. Cette dernière, mis à part le contexte qu'elle fournit à l'objet de cette étude, met en place le cadre administratif dans lequel s'exercera pratiquement la stérilisation des malades mentaux. 
Examinons pour commencer l'établissement des responsabilité dans l'application des textes. Ce qui frappe de prime abord, c'est l'imprécision des attributions du Conseil de santé, le contrôle plutôt lâche des pratiques, caractéristique de l'indépendance laissée à l'administration et aux médecins à l'intérieur du cadre législatif. On ne donne que de très vagues lignes directrices, que les hommes chargés de l'application pourvoiront de sens. Il y a bien sûr une explication. Si l'on s'attarde sur la composition de la commission préparatoire, on s'aperçoit que l'administration de la santé y a la majorité absolue, avec 8 membres sur 13. Logiquement, la commission a voulu imposer un minimum de contraintes à l'exercice quotidien d'une loi sanitaire qu'une bonne partie de ses membres seront chargés d'appliquer.

L'importance de ces quelques points sur l'objet de notre exposé est énorme, car c'est le Conseil de santé qui aura le dernier mot sur les stérilisations des malades mentaux. La procédure prévue est en effet la suivante: le tuteur, la famille, l'autorité communale ou municipale responsable saisissent le Conseil de santé d'un cas, en envoyant une déclaration médicale portant diagnostic. Le Conseil tranche. S'il trouve l'intervention justifiée, il nomme trois experts, d'habitude deux psychiatres et un gynécologue, qui doivent rendre un rapport concordant. Les experts donnent le type de la maladie, l'incurabilité ou non du cas, la probabilité des tares qui affecteront la descendance potentielle. Le Conseil, après examen des rapports, prend la décision finale. Son «autorisation» est donc primordiale.

\section{Maladie mentale: ses images et ses acceptions}

Si l'on essaie de cerner d'un peu plus près les actions du Conseil de santé et des experts, on s'aperçoit très vite que l'autorité politique se décharge de toute responsabilité au profit des «professionnels» de l'administration de la santé et des médecins. Les décisions de stérilisation sont comprises comme devant être le fruit exclusif des délibérations de ces derniers. Le choix des motifs leur appartient, et, a priori, aucun n'est vraiment exclu ${ }^{11}$. Pour saisir les conséquences d'une telle liberté, il faut se pencher sur les figures de la maladie mentale, sur ses images dans les esprits. Or, pour les aliéniștes et les hommes politiques, il ressort avant tout que le malade est une figure dont les coûts sont exorbitants, qu'ils soient sociaux ou matériels.

Il y a d'abord la peur, courante partout en Europe à cette époque, de l'accroissement géométrique des vagabonds, des malades mentaux, des 
prostituées, des alcooliques et des criminels. Elle découle avant tout d'une amélioration des outils statistiques et d'une augmentation de l'offre de soins. A l'époque, on y a pourtant plutôt vu la manifestation d'une sexualité débordante et peu contrôlée, qui a pour conséquence que les individus les plus «indésirables» se reproduisent le plus. C'est le fameux concept des différentiels de fécondité, qui voulait que plus un membre était socialement utile à la société, moins il avait d'enfants. Les classes les moins responsables, se fichant éperdument des conséquences d'une conception, copulaient fréquemment, augmentant ainsi le nombre d'individus peu doués, le plus souvent constitutionnellement tarés.

La peur de cet accroissement, quand il concerne le malade mental, s'applique avant tout au faible d'esprit, souvent considéré comme majoritaire dans la population des malades mentaux. Ces «faibles d'esprit», à l'appellation très générique, ce sont

«[...] les idiots, les imbéciles et les débiles mentaux et moraux de tous les degrés. On appelle globalement tous ces insuffisants congénitaux des oligophrènes.» ${ }^{12}$

C'est à travers l'oligophrène que s'opère la jonction entre la maladie mentale et ses figures anti-sociales. C'est sous cette rubrique que seront classées plus du $9 / 10^{\text {e }}$ des stérilisations pratiquées. Le Dr. Steck, de Cery, lie par exemple très expressément l'oligophrène à une menace sociale:

«L'importance sociale de ce chiffre [celui du nombre des oligophrènes en Suisse] augmente encore lorsqu'on sait que c'est dans cette catégorie d'anormaux que se recrute un grand nombre d'anti-sociaux et de criminels. La criminalité des faibles d'esprit est beaucoup plus grande que la criminalité des schizophrènes par exemple. C'est parmi les faibles d'esprit que se recrutent les vagabonds, les prostituées, les assistés de toute sorte, les profiteurs d'assurance.»

La menace est transmissible:

«Un grand nombre, même la majorité des faibles d'esprit sont des dégénérés héréditaires.» ${ }^{13}$

Les députés, et ce n'est pas surprenant, s'attardent tout particulièrement sur le coût financier de ces oligophrènes. Comme la plupart des aliénistes, ils sont persuadés que la maladie mentale, la «tare», est héréditaire. Elle a aussi le défaut de coûter cher:

«[...] j'ai pu constater que les familles dégénérées se suivent de génération en génération et, si l'on pouvait supprimer cette descendance anormale, ce serait un bien pour la société!» «[...] au point de vue pratique nous avons déjà vu des cas où l'on a procédé à la stérilisation 
d'assistés de la commune, de filles, qui étaient des filles-mères, et qui pendant toute leur vie ont coûté à la commune; si nous n'avions pas procédé à la stérilisation, l'enfant aurait aussi coûté pendant toute sa vie.» ${ }^{14}$

L'opposition à l'article $28^{\text {bis }}$ est très maigre. Elle émane avant tout des rangs du parti libéral qui la justifie pour des raisons éthiques. On trouve qu'une telle mesure, importée de l'étranger, a des relents de «matérialisme grossier». On remarque aussi que les lois de la «transmission des tares» sont assez nébuleuses. L'article $28^{\text {bis }}$ sera cependant voté, comme le reste de la réforme, à une très grande majorité.

La presse, mise à part la libérale Gazette de Lausanne, restera relativement silencieuse. Le sujet n'intéresse pas beaucoup l'opinion. C'est dans un même climat d'indifférence polie qu'auront lieu les débats de 1931 sur la révision du code pénal vaudois. A l'avortement eugénique légal qui en découle, on attribue le même intérêt qu'à la stérilisation, et il est faible.

\section{La Société de patronage des aliénés du canton de Vaud}

Ce climat de consensus entre le parlementaire et le scientifique, au milieu de l'indifférence des médias, demande à être expliqué. C'est qu'entre l'existence d'un thème, sa popularité et sa diffusion, il est nécessaire généralement d'avoir des diffuseurs qui rendront les variables du dit thème compréhensibles et attractives.

Que l'hérédité des tares mentales soit une idée extrêmement répandue ne lui donne pas pour autant un accès direct au politique. Il faut que s'y ajoutent à la fois l'impression de détenir un remède et le sentiment d'une menace. Il faut enfin qu'un groupe de pression ou des individus motivés agissent sur les instances de prise de décision.

La menace, on l'a dit, est ressentie. La multiplication exponentielle d'individus tarés et anti-sociaux est un thème qui revient comme un leitmo$t i v$ dans les sources. Les remèdes sont fixés depuis longtemps: ségrégation stricte des aliénés dans les asiles, mesures sur les mariages, consultations médicales avant l'hymen ${ }^{15}$, stérilisation. Toutes ces mesures ont déjà été appliquées à l'étranger. Mais si l'on saisit le mal, encore faut-il populariser les remèdes, justifier une intervention coercitive qui serait mise en place par une loi. Ce travail de lobbying sera effectué dans le canton de Vaud par la Société de patronage des aliénés, satellite de l'Hôpital de Cery. ${ }^{16}$ 
Cette dernière naît au début du siècle à Lausanne, sous l'égide de la Ligue pour l'action morale. Les buts de la Société tels qu'ils sont définis par le docteur Mahaim de Cery reprennent le vieil impératif de protection. Il s'agit tout d'abord de fournir une aide au malade, essentiellement matérielle, lors de sa réinsertion. Il faut aussi lutter contre les préjugés qui entourent le malade, répandre les données exactes qui doivent transformer dans l'opinion le fou en un malade, dont l'état nécessite des soins attentifs donnés par des spécialistes.

La Société veut aussi rendre le public attentif aux menaces qui pèsent sur l'hygiène mentale générale, par l'instruction ou par la lutte contre les produits qui engendrent des effets négatifs pour la santé mentale.

Dans ce travail d'instruction et de propagande, la Société a besoin de l'Etat. La santé mentale et sa prophylaxie englobent de nombreux domaines, dont seul le souverain peut régler les modalités particulières. Dès le début, la Société a insisté sur le rôle actif qu'elle entendait jouer au niveau politique dans les domaines relevant de l'hygiène mentale. Elle est et se reconnaît comme un groupe de pression. Les statuts le disent très explicitement.

L'importance de la «voie gouvernementale» dépend de l'autorité et du prestige de la Société, des relais dont elle dispose dans le monde politique, économique et social. Vu sous cet angle, son travail a été efficace. Trois sondages ont été effectués, en 1923, 1928 et 1936. Les membres collectifs m'ont tout d'abord intéressé: les municipalités, communes ou conseils de paroisse représentent entre treize et seize pour-cents de l'effectif total de la Société. En chiffres absolus, cela fait plus de 70 communes ou conseils. On peut donc dire que la Société dispose de bonnes antennes dans le canton, auxquelles on peut ajouter l'influence qu'exercent dans les localités le grand nombre de pasteurs et d'instituteurs qu'elle compte dans ses rangs.

La place des spécialistes, qu'ils soient médecins ou plus généralement universitaires, est aussi très importante, mais plus difficilement chiffrable, en raison de l'imperfection des listes. On pourra observer que l'organigramme de la Société suit de très près celui de Cery, et qu'une promotion ou un départ dans l'un est toujours accompagné d'un mouvement équivalent dans l'autre. A tel point qu'il est facile de voir dans la Société de patronage des aliénés une dépendance de Cery.

Au niveau politique, on retrouve la trace de la Société à l'intérieur même de la commission législative chargée de la révision de la loi sanitaire de 1897. Deux membres sur les treize de la commission, et pas les moins prestigieux, 
font partie de la Société. Il s'agit d'un juge fédéral et d'un docteur. Un des principaux rédacteurs de l'article $28^{\text {bis }}$ nouveau sur les stérilisations est inscrit dans une société sœur de la Société de patronage des aliénés, le comité «Question sexuelle» de la Ligue pour l'action morale. Des membres du Conseil de santé sont tout autant membres de la Société.

L'importance de ces antennes dans le monde politique est grande parce qu'elles véhiculent les prises de position de la Société, visibles par l'intermédiaire des rapports annuels. Là encore, à leur lecture, une image très nette se dégage, c'est l'importance accordée aux facteurs héréditaires dans le déclenchement des maladies mentales. Il peut y avoir certains différends quant au rôle exact des déterminants héréditaires, mais leur importance n'est jamais remise en cause. La menace que peuvent constituer des individus mentalement ou physiologiquement tarés pour le corps social est prise très au sérieux. C'est ainsi que la Société en vient à défendre l'idée de la stérilisation des malades mentaux comme moyen de réduire le nombre d'individus porteurs d'hérédités malsaines ou dangereuses. Forel en parle dès 1904 dans le rapport annuel de la Société, où il envisage aussi la probabilité d'une forme d'euthanasie pour les malades les plus atteints. Les docteurs Mahaim (1921), Boven (1928), Steck (1937, 1944) reprendront le thème de la stérilisation des malades mentaux. Ils le feront également dans d'autres articles publiés dans les revues de psychiatrie ou d'utilité publique de l'époque.

Les prises de position de la Société de patronage importent quand on saisit les connexions dont elle dispose. On a retrouvé des membres de la Société dans la commission législative, lors des consultations, dans le Conseil de santé (deux membres), dans les communes qui ont autorité de saisir ce même Conseil d'un cas, et on va retrouver les plus prestigieux d'entre eux parmi les experts nommés par le Conseil de santé selon la procédure prévue pour l'application de l'article de loi. Où que se tourne le regard en 1928, il heurte la Société de patronage des aliénés.

\section{L'application du texte}

La vision passablement négative du malade mental qui traverse le débat et l'intervention des spécialistes vont immanquablement revenir dans les applications pratiques du texte. La figure de l'oligophrène menaçant et trop fécond se retrouve tout à fait dans les bilans chiffrés, hélas lacunaires, qui ont 
été publiés. Dans une proportion écrasante, de l'ordre des $9 / 10^{\mathrm{e}}$, ce sont des oligophrènes de sexe féminin qui ont été stérilisées ${ }^{17}$. Encore faudrait-il savoir ce que l'on entend par «oligophrène» et s'il n'est pas possible de déterminer plus exactement comment et pourquoi la loi a été appliquée.

Pratiquement, l'oligophrénie est très dure à définir. On parle d'idiots, d'imbéciles, de débiles mentaux et moraux de tous les degrés. Hans Steck relevait, et il n'était pas le seul à le faire, les liens entre cette classe de malades et nombre de comportements anti-sociaux qui allaient du crime à la prostitution en passant par le goût des narcotiques. Ils sont aussi pourvus d'une sexualité débordante.

La variable comportementale, surtout dans sa variante sexuelle, me semble la plus importante pour caractériser les oligophrènes. La particularité de ces infortunés, c'est qu'ils vivent cachés, immergés dans le reste de la population jusqu'à ce qu'un acte révèle leur tare. Etant plus légèrement atteintes que les cas internés, ils vivent dans le corps social, au sein duquel ils exercent une influence souvent corruptrice.

L'illégitimité des naissances a dans cette optique un lien très fort avec l'oligophrénie. On peut dire que ce révélateur de conduites sexuelles hors cadre dénonce l'oligophrénie pour au moins deux raisons. La première, c'est que l'oligophrène, ne pouvant assumer les devoirs moraux qui découlent de la procréation, copule sans retenue et le plus souvent hors mariage. C'est à cette activité sexuelle passionnée que l'on attribue la montée exponentielle de la maladie mentale et de la déviance en général. La seconde raison d'un tel lien entre faiblesse d'esprit et illégitimité, c'est que les enfants nés hors mariage fournissent le plus fort contingent, croit-on, de la population carcérale et asilaire ${ }^{18}$. Leur lien avec le crime et la folie est tenu pour certain. Ce fait, qui est peut-être juste, statistiquement parlant, n'est à l'époque expliqué par aucune considération sociale dans la littérature aliéniste. L'hérédité malheureuse est seule mise en avant.

Triple malédiction, l'illégitimité remplit les asiles et les prisons, révèle les conduites sexuelles hors-norme, et se montre un vecteur par lequel les individus transmettent leurs tares à des descendants que l'on imagine multiples.

Les conséquences de cette relation entre l'illégitimité, symbole d'une sexualité hors-norme, et l'oligophrénie, sont capitales dans l'application pratique de l'article $28^{\text {bis }}$. Tous les témoignages révèlent en effet que la motivation principale d'une commune, quand elle saisit le conseil de santé d'un cas, est économique. 
Il s'agit le plus souvent d'une femme pauvre qui a des enfants assistés, des grossesses hors mariage ou les deux ${ }^{19}$. La logique de la commune est ici d'exploiter la possibilité offerte d'une stérilisation sous le nouveau cadre législatif. Cette demande à motif économique précède les travaux du Conseil de santé.

Il reste encore, à ce niveau de la procédure, un lien logique à établir. Pour que s'exerce pleinement l'article $28^{\text {bis }}$, il faut établir une relation entre des comportements sexuels ressentis comme déviants ou gênants et une maladie mentale. La notion de «catégorie perverse» va nous permettre de jeter des ponts. Elle a été développée par Ferdinand Morel, mais ce genre de catégorisation est loin de lui être propre. Une lecture approfondie des sources met à jour l'utilisation de tels concepts, au moins implicites.

Rappelons que la définition de l'oligophrénie inclut les idiots, les débiles mentaux et moraux, les imbéciles qui sont des «insuffisants congénitaux». $\mathrm{Si}$ on se place sur le terrain de la constitution, qui représente l'individu dans la totalité de ses éléments héréditaires, la constitution perverse regroupe tous les débiles mentaux et moraux congénitaux dont le comportement donne lieu à «des conflits répétés avec la morale». L'oligophrène donc, s'il a un comportement qui va contre la morale, peut devenir une «constitution perverse». Le contraire va hélas être aussi vrai : le comportement qui donne lieu à des conflits répétés avec la morale sera compris pratiquement comme révélant l'oligophrène, acte-vérité qui le débusquera de sa niche. La relation logique et diagnostique entre le malade et son acte va se retourner, s'inverser. C'est la présomption de maladie qui va découler de l'acte immoral, alors que le second n'aurait dû être expliqué que par le premier ${ }^{20}$. Ce passage à la réflexion entre les deux termes de la proposition «catégorie perverse» découle du flou constant qui est entretenu entre les figures du malade, de l'anti-social et du toxicomane.

C'est dans cette optique que l'on peut interpréter la forte proportion de femmes oligophrènes dans le total des stérilisations pratiquées. Les grossesses illégitimes révèlent une sexualité hors norme, remplissent les prisons et les asiles et donnent lieu à un conflit avec la morale qui est symptomatique de l'oligophrène. En stérilisant la coupable ainsi reconnue, en l'avortant parfois pour faire bonne mesure, on empêche qu'elle ne reproduise sa tare, on limite les frais directs et indirects que l'on impute à sa future progéniture. 


\section{Conclusion}

Il y a parfois de singulières prémonitions. Au plus haut de l'hystérie sur le «péril vénérien», un jeune médecin exposait son credo, tout en regrettant son caractère utopique:

«Formulons nettement l'idéal vers lequel doivent tendre nos efforts: empêcher d'entrer en union conjugale tout homme incapable d'avoir une descendance bien portante et cela sans léser aucun droit humain. [...] Le seul moyen vraiment efficace consiste dans la surveillance incessante et autoritaire, dans le sens le plus large du mot, de tout individu atteint d'une maladie contagieuse et héréditaire.» ${ }^{21}$

Beaucoup ont alors partagé ce fantasme de la résolution de la question sociale par la science ${ }^{22}$. Faut-il rappeler cependant qu'au-delà des aspects les plus percutants des débats, des jeunes femmes ont été opérées ou avortées contre leur volonté, sous la menace d'un internement ou grâce aux «amicaux conseils» de communes intéressées? Ces souffrances-là sont silencieuses, hélas.

«Mais ces ombres, qui étaient lasses et nues, changèrent de couleur et claquèrent des dents dès qu'elles entendirent ces mots cruels. Elles blasphémaient Dieu et leurs parents, l'espèce humaine et le lieu et le germe de leur naissance, et de leur lignée.» (Dante, Inferno, III 95-105, trad. J. Risset, Paris, 1985.) 
Notes

1 Pour la position, très critique, de l'Eglise catholique: L'encyclique sur le mariage chrétien Casti connubii, traduction française avec divisions et commentaires, Paris, SPES, 1931.

2 Nisot M. T., La question eugénique dans divers pays, vol. 1, Bruxelles 1927. Jichlinski S., Les stérilisations et castrations eugéniques, prophylactiques et thérapeutiques, Genève, thèse de médecine, 1941. Steck Hans, «La pratique de la stérilisation légale des anormaux psychiques dans le canton de Vaud», in Revue médicale de la Suisse romande, LV-13 (1935), pp.874-7; même auteur, «Les dispositions légales concernant la stérilisation eugénique dans le canton de Vaud et leur application», Vierter Jahresbericht der Schweizerischen Gesellschaft für Vererbungsforschung, (1944), pp.481-5. Sutter Jean, L'eugénique, Paris, 1950. (Institut national d'études démographiques, Travaux et documents, cahier No 11.)

3 Canton de Vaud, loi du 27 mars 1906 sur l'internement des alcooliques. Bulletin des séances du Grand Conseil du Canton de Vaud, Lausanne, 1901, 1921 et 1928. Recueils des lois et décrets du canton de Vaud, mêmes années. Débats sur la stérilisation des malades mentaux, 1928, pp. 507-539, 897-916; en annexe «Exposé des motifs du projet de loi remplaçant la loi du 14 septembre 1897 sur l'organisation sanitaire.» Séances des 3 mars et 12 mai 1931 : débat sur l'avortement eugénique; séance du 23 mai 1939: modification refusée du texte de 1928.

4 Dizard F., Etude sur le morphinisme et son traitement, Genève, 1893. Morel Ferdinand, Introduction à l'hygiène mentale, s.l.n.d. (Conférence faite à l'assemblée romande du Cartel romand d'hygiène sociale et morale, Lausanne, 6 octobre 1932.)

5 Note (3).

6 «Exposé des motifs du projet de loi remplaçant la loi du 14 septembre 1897 sur l'organisation sanitaire», op. cit. Bulletin des séances, (1928), pp.514-6.

7 Ruggli Paul, Les résultats de la castration thérapeutique sur les aberrations sexuelles, Genève, Thèse de médecine, 1943.

8 Ehrenström Philippe, «La stérilisation des malades mentaux», in Médecine et hygiène, 48 (1990), pp.2786-2790.

9 Ehrenström Philippe, «Regards aliénistes dans les cantons de Vaud et Genève de la fin du XIX $^{\text {e }}$ siècle aux années 1930», in Equinoxe, 3 (été 1990), pp. 169-178.

10 Société de patronage des aliénés du canton de Vaud, (abrégé SPA) 4*, 20 et $27^{\circ}$ rapports, Lausanne, 1904, 1922 et 1929.

11 Bulletin des séances, (1928), pp. 513-4. Souligné par moi.

12 Morel Ferdinand, op. cit., p.2.

13 Steck Hans, op. cit., RMSR (1935), p. 881. Même auteur: «Eugénisme et stérilisation», in SPA, 35" Rapport année 1936, Lausanne, 1937, p. 13; "Quelques considérations sur l'hygiène mentale», in SPA, 42e Rapport année 1943, Lausanne, 1944, p. 5.

14 Bulletin des séances, (1928), p.537.

15 Kamler Antoine, Le certificat médical avant le mariage, Genève, Thèse de médecine, 1911. Krafft H. C., «Consultations médicales matrimoniales», in Revue suisse d'hygiène mentale, 12 (1932), pp.454-8. Même auteur, «Consultations médicales de mariage de Pro Familia au dispensaire de La Source, Lausanne», in Revue suisse d'hygiène, 13 (1933), pp. 232-4.

16 Les archives de la Société de patronage des aliénés sont déposées au département des manuscrits de la bibliothèque de Dorigny, cote IS 3696 . 
17 Voir les articles du Dr. Steck, note (2).

18 Deux exemples: Ladame P., Des enfants illégitimes en Suisse, Lyon, 1882; «L'hypnotisme et la pédagogie», in Revue de l'hypnotisme, 1 (1887), p. 367.

19 Voir les cas publiés par Wanner François, «La loi sur la stérilisation des personnes privées de discernement et son fonctionnement dans le canton de Vaud pendant la première année», in L'hygiène mentale, 25 (1930), pp. 170-1.

20 Un exemple dans Naville F., «Etudes sur les castrations et les stérilisations thérapeutiques et prophylactiques en médecine sociale et en psychiatrie», in Revue médicale de la Suisse romande, XLV (1925), pp.622-3.

21 Kamler A., op. cit., p. 15.

22 Le lecteur intéressé pourra se reporter au mémoire de licence sur l'eugénisme et la question sociale, que j'ai soutenu à la Faculté des lettres de l'Université de Genève en juillet 1989. 


\section{Summary}

\section{Surgical sterilization and insanity}

The author examines the intellectual, social and political conditions which permitted the introduction of compulsory sterilization of mentally ill as a lawful measure in the Canton of Vaud in 1928. Essential was the fear of «degenerated» and socially harmful progeny prejudicial to the existing order. The victims of this regulation were mostly feeble-minded women-the poorest and least respected members of community.

\section{Zusammenfassung}

\section{Operative Sterilisation und Geisteskrankheit}

Der Autor stellt das geistige, soziale und politische Umfeld des waadtländischen Zwangssterilisations-Paragraphen von 1928 dar. Dessen Einführung gründete auf der Furcht vor degenerierter und sozial schädlicher Nachkommenschaft, die als Bedrohung der bestehenden Ordnung angesehen wurde. Vor allem schwachsinnige Frauen - die Ärmsten und Verachtetsten - wurden Opfer dieser Massnahme.

Philippe Ehrenström

lc rue Beau-Site

1203 Genève 